\title{
Determination of carcinogen 4,4'-diamino diphenyl methane (MDA) in polyols derived from flexible polyurethane foam solvolysis by combination of SEC and GC/MS
}

\author{
Eckhart Kornejew, Valentin Stoychev
}

Zusammenfassung

Vorgestellt wird eine Methode zur quantitativen Bestimmung der krebserregenden Substanz 4,4'-Diaminodiphenylmethan (MDA) in Polyolen, die aus PolyurethanWeichschäumen durch Solvolyse hergestellt wurden.

Dabei wird zunächst der niedermolekulare Anteil mittels Gelpermeationschromatographie abgetrennt und enthaltenes MDA anschließend über Gaschromatographie/ Massenspektrometrie bestimmt.

\begin{abstract}
A method is presented to determine the concentration of the carcinogen 4,4'-diamino diphenyl methane (MDA) in polyols derived from polyurethane flexible foam solvolysis by combination of size exclusion chromatography (SEC) and gas chromatography coupled to mass spectrometry (GC/MS).

The first step of the method is the separation of the MDA containing fraction by SEC while the second step consists in the quantitative analysis of the MDA amount by GC/MS.

Quantitation limits of the method are lower than $0.05 \%$ MDA in the polyol samples with an accuracy of $\pm 12 \%$ of the determined values.
\end{abstract}

\section{Introduction}

Polyurethanes (PUR) of any kind may be treated with glycols and/or amines to obtain polyol products, which can be introduced into polyurethane formulations again [1] (Behrendt et. al. 2009). This process called PUR solvolysis, in particular glycolysis and aminolysis, can be applied to industrial wastes of PUR and represents the major way of chemical recycling in this branch [2] (Behrendt et. al. 2005: 85-92), [3] (Raßhofer 1995), [4] (Thor et. al. 2005), [5] (Parrinello et. al. 1997), [6] (Kierkus et. al. 1998), [7] (Gassan et. al. 1992).

The PUR solvolysis, i.e. the cleavage of the urethane bond, leads mainly to polyols, $\omega$-(hydroxyalkyl)urethanes, and poly urea compounds. During the solvolysis of polyurethanes based on aromatic diisocyanates side reactions occur, one of which is the formation of primary aromatic diamines [1] (Behrendt et. al. 2009). In the case of polyurethanes produced with 4,4'-diphenyl methane diisocyanate (MDI) the resulting aromatic diamine is $4,4^{\prime}$-diamino diphenyl methane (MDA). 4,4'-MDA is considered as a Class IIA carcinogen [18] (WHO, IARC Monographs 57: 1993) and therefore subject to certain restrictions. Thus, the maximum amount of MDA in substances to be shipped is limited to $0.1 \%$ by European legislation [19] (REGULATION (EC) No 1272/2008).

Hence it is very important to know the concentration of MDA in polyols derived from PUR solvolysis. Depending on the results it is usually necessary to reduce the MDA level by means of deamination [4] (Thor et. al. 2005) to meet the legal requirements.

$4,4^{\prime}$-MDA is a white to yellowish solid compound having a melting point of $92^{\circ} \mathrm{C}$ and a boiling point of $398^{\circ} \mathrm{C}$. As a result of the technical grade $4,4^{\prime}$-MDI used in the PUR production, which always contains smaller amounts of 2,4'-and 2,2'-MDI isomers, the MDA formed during the PUR solvolysis is consequently a mixture of three isomers as well: 


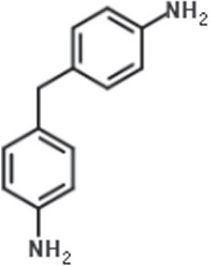

4.4'-MDA

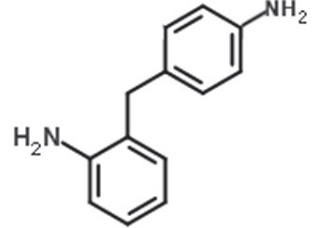

2.4'-MDA

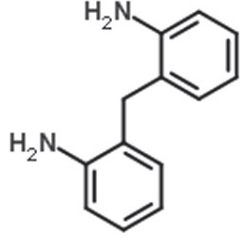

2,2'-MDA figure 1: main isomers of MDA

Analytical methods for the determination of MDA have been developed for several purposes. A large part of it relates to the analysis of MDA in environmental samples or liquids like urine or blood plasma from workers exposed to MDI, using the MDA as a bio marker for MDI [9] (Skarping et. al. 1995), [10] (Neumeister 1994).

The instrumental equipments used for the determination of MDA are HPLC, LC/MS, or GC/MS [11] (Shintani et. al. 1989), [12] (Mattrel et. al. 1995), [13] (Mazzu et. al. 1997). In some cases the GC/MS analysis is preceded by a derivatization of MDA [14] (Schmidt et. al. 1997).

The method described here was developed with the objective to be sensitive and accurate enough to determine an MDA amount in the polyol samples lower then $0.1 \%$, and furthermore, to attain an easily applicable method without special sample preparation or derivatization by combination of SEC and GC/MS.

\section{Experimental}

\subsection{Polyol preparation}

The PUR based polyols were obtained by aminolysis of high resiliency foam (F.S. Fehrer Automotive $\mathrm{GmbH}$ ) using diethylene triamine and N(2)-methyl diethylene triamine as reagents. After the reaction was considered to be complete the upper liquid polyol phase was separated from the lower precipitated poly urea phase [8] (Stoychev et. al. 2006). When choosing only amines as solvolysis reagents the reaction products are polyol(s) and oligo ureas with unknown amounts of MDA accompanying the polyol(s) only.

\subsection{Size Exclusion Chromatography (SEC)}

Size Exclusion Chromatography (SEC), also known as Gel Permeation Chromatography (GPC), is a method to separate molecules in solution based on their hydrodynamic volume, i.e. the size of a molecule combined with its shape [15] (Mori 1999).
In our method developed, the SEC is used to isolate the MDA containing fraction of low molecular size from the higher molecular size polyol.

\section{Device configuration}

The analytical SEC device manufactured by Viscotek and connected to a ViscoGEL GMH HR-N column was run with tetrahydrofuran (THF) as the solvent at a flow rate of $0.8 \mathrm{ml} / \mathrm{min}$ and an injection volume of $100 \mu \mathrm{l}$. For detection only the RI signal was employed and interpreted using the Software OmniSEC v4.2.

\section{Range of fractionation}

To fractionate the eluent its delay to cover the distance between analyzer and outlet had to be determined. This was simply done by injecting a solution of phenolphthalein and observing the color change when the eluent dropped into a potassium hydroxide solution which continuously flowed through a slightly inclined glass tube. Thus, the difference between the start of appearance of the phenolphthalein peak and the beginning of color change was measured as well as the delay from the end point of the peak until total clearance of the eluent/KOH stream.

A solution of pure 4,4'-MDA provided by courtesy of Performance Chemicals Handels $\mathrm{GmbH}$ was injected to determine the range the eluent flow had to be cut within. This range of fractionation was broadened by $0.2 \mathrm{ml}$ in both directions to ensure the capture of all MDA contained in a sample.

\section{Reference samples}

To calibrate the method a series of reference samples was prepared by mixing a polyol with different quantities of 4,4'-MDA, ranging from $0.05 \%$ to $10 \%$, and dissolving the mixture in THF (each sample $50 \mathrm{mg} / \mathrm{ml}$ ). The polyol used was Lupranol ${ }^{\circledR} 2095$, a trifunctional standard polyether polyol with primary hydroxyl groups, provided by courtesy of Elastogran $\mathrm{GmbH}$.

As the concentration of a sample injected to the analytical SEC device is limited to $50 \mathrm{mg} / \mathrm{ml}$ not to overload the column, the resulting vial concentration of MDA in THF is $50 \mu \mathrm{g} / \mathrm{ml}$ in case of a reference sample containing 0.1\% MDA. Thus the total MDA amount in the eluent fraction after injecting $100 \mu$ l of this sample equals only $5 \mu \mathrm{g}$. Therefore each sample was injected two times and the separated eluent portions joined together to increase the amount of analyzable MDA and minimize the margin of error. 


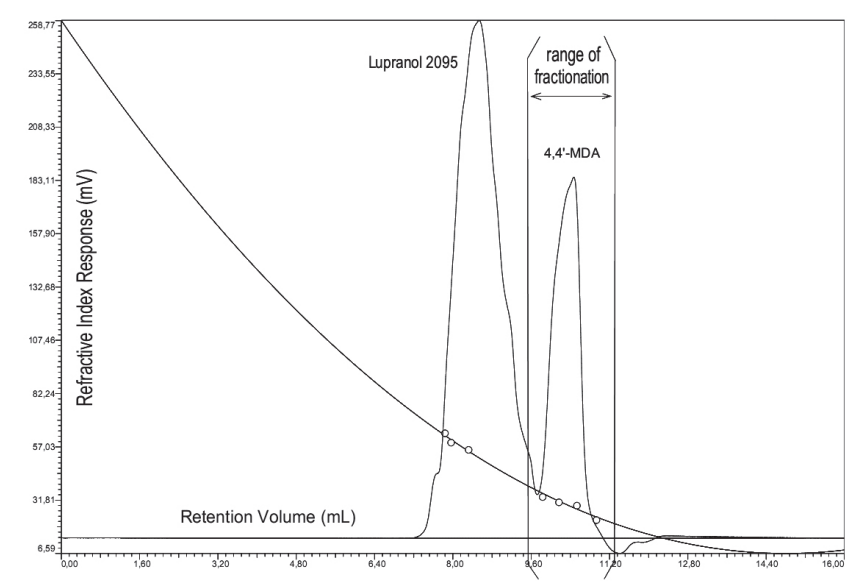

figure 2: size exclusion chromatogram of $10 \%$ 4,4'-MDA in Lupranol 2095 with the range of fractionation marked

\section{Polyol sample run}

The polyol samples obtained by PUR aminolysis were treated in the same manner: dissolved in THF (50 mg/ $\mathrm{ml})$, injected twice and the separated eluent portions joined.

\subsection{Gas Chromatography/Mass Spectrometry (GC/MS)}

\section{Device configuration}

The GC device applied in this method was a HP 5890 series II with a ZEBRON ZB-35 capillary column of intermediate polarity, helium as carrier gas adjusted to a column head pressure of $60 \mathrm{kPa}$ and an injection split ratio of ca. 10:1. The temperature of the injector was set to $300{ }^{\circ} \mathrm{C}$ as well as the initial oven temperature, which after injection followed a heating rate of $10 \mathrm{~K} / \mathrm{min}$ up to a final temperature of $400^{\circ} \mathrm{C}$.

The attached MS was a HP 5970 series device with a quadrupole detector, set to start with scanning at 3 minutes after injection.

The Software used was MS ChemStation and NIST MS Search v2.0 (175K spectra).

\section{Reference sample run and calibration curve}

The solvent of the SEC derived eluent fractions of each reference sample was evaporated at $60{ }^{\circ} \mathrm{C}$. Next, the residue was dissolved in $200 \mu \mathrm{l}$ THF.

$10 \mu \mathrm{l}$ of any of the calibration samples prepared in this way were injected into the gas chromatograph using five runs for each sample. Based on the MDA concentrations of the original reference samples before SEC and the average values of the resulting GC peak areas a calibration curve was calculated (table 1, figure 3).

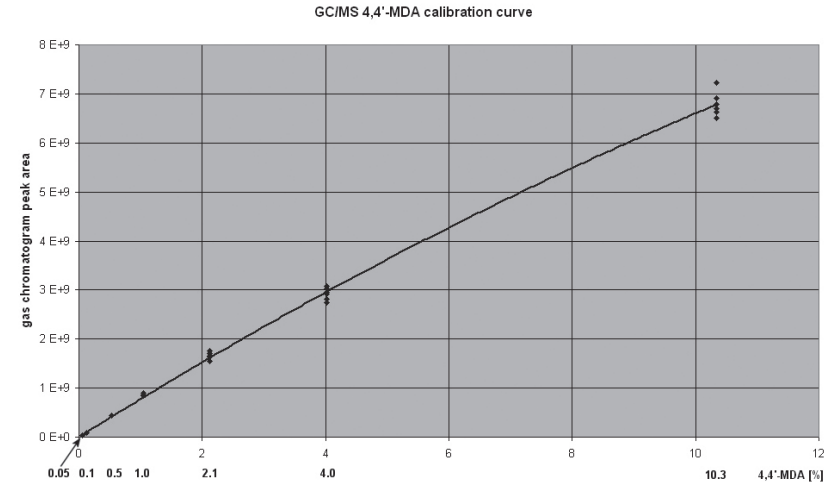

figure 3: GC/MS 4,4'-MDA calibration curve

\section{Polyol sample run}

The SEC derived eluent fractions of the polyol samples were treated in the same way as the reference samples: solvent evaporated at $60{ }^{\circ} \mathrm{C}$, residue dissolved in $200 \mu \mathrm{l}$ THF and $10 \mu \mathrm{l}$ of each vial injected several times.

\section{MDA isomers}

As mentioned above, the MDA formed during the PUR foam solvolysis usually is a mixture of 4,4'-MDA as the main component and smaller amounts of 2,4'- and 2,2'-MDA isomers.

Figure 4 shows the gas chromatogram of a polyol sample with 4,4'-MDA as the largest peak and two smaller ones, which could clearly identified as being

\begin{tabular}{|c|c|c|c|c|c|c|c|c|}
\hline \multicolumn{9}{|c|}{ 4,4'-MDA calibration data } \\
\hline \multirow[t]{2}{*}{$C_{M D A}[\%]$} & \multicolumn{5}{|c|}{ peak area $\mid 10^{\wedge} 6$} & \multicolumn{3}{|c|}{ statistics } \\
\hline & inj. № 1 & 2 & 3 & 4 & 5 & $\mu$ & $\sigma$ & $\mathrm{Cv}$ \\
\hline 0,05 & 27,8 & 28,6 & 26,7 & 28,1 & 26,8 & 27,6 & 0,8 & 0,030 \\
\hline 0,12 & 93,8 & 89,5 & 90,4 & 91,2 & 93,4 & 91,7 & 1,9 & 0,020 \\
\hline 0,54 & 442,3 & 441,2 & 444,0 & 438,7 & 442,1 & 441,7 & 1,9 & 0,004 \\
\hline 1,05 & 838,6 & 888,0 & 843,6 & 863,0 & 850,4 & 856,7 & 19,7 & 0,023 \\
\hline 2,13 & $1.707,6$ & $1.642,6$ & $1.538,5$ & $1.750,5$ & $1.649,6$ & $1.657,8$ & 80,0 & 0,048 \\
\hline 4,02 & $3.077,7$ & $2.955,4$ & $3.020,4$ & $2.805,0$ & $2.739,6$ & $2.919,6$ & 143,2 & 0,049 \\
\hline 10,34 & $6.627,5$ & $6.692,5$ & $6.500,5$ & $7.222,0$ & $6.908,0$ & $6.790,1$ & 283,0 & 0,042 \\
\hline
\end{tabular}

table 1: 4,4'-MDA concentrations and GC peak areas 
isomers of MDA. Unfortunately, the mass spectra of both isomers did not sufficiently match the library records, and reference samples of the MDA isomers were not available. Thus, an explicit identification of isomer $\mathrm{A}$ and $\mathrm{B}$ was not possible. Considering the isomer distribution in technical grade MDI, which is a result of different chemical reactivities of the carbon atoms in a substituted aromatic ring during the synthesis process, it can be assumed that isomer A is 2,2'-MDA and isomer B 2,4'-MDA. This is supported by the distribution of MDI isomers in liquid MDI which consists of approximately $54 \mathrm{wt} \%$ of the $4,4^{\prime}$-isomer, $45 \%$ of the $2,4^{\prime}$-isomer, and about $1 \%$ of the 2,2 '-isomer (first fraction of pure MDI distillation).

\section{Detection limit}

To determine the limit of detection (LOD), which in GC/MS approximately is the concentration of substance where the signal response is three times the background noise, several injections with lower MDA concentrations were made. Thus, a detection limit of approximately 2 ng 4,4'-MDA per $\mu$ THF was found.

\section{Results}

Seven samples of the high resiliency PUR foam derived polyols were analyzed resulting in the following 4,4'MDA concentrations:

\begin{tabular}{|l|r|c|c|c|c|c|c|c|c|}
\hline \multirow{2}{*}{ sample } & \multicolumn{4}{|c}{ CMDA [\%] } & \multicolumn{4}{c|}{ statistics } \\
\hline inj. № 1 & 2 & 3 & 4 & 5 & $\mu$ & $\sigma$ & CV \\
\hline VSA14 & 7,0 & 6,9 & 7,1 & 6,6 & 7,2 & 7,0 & 0,19 & 0,03 \\
\hline VS14ACAC & 1,7 & 1,6 & 1,9 & 1,7 & 1,8 & 1,7 & 0,10 & 0,06 \\
\hline VS127 & 6,3 & 5,9 & 6,2 & 5,8 & 5,7 & 6,0 & 0,22 & 0,04 \\
\hline VSA & 4,3 & 4,4 & 4,8 & 4,3 & 4,7 & 4,5 & 0,22 & 0,05 \\
\hline VSA+50 & 2,9 & 3,0 & 3,2 & 3,4 & 3,1 & 3,1 & 0,17 & 0,06 \\
\hline VS113-1+GE & 0,7 & 0,6 & 0,7 & 0,8 & 0,7 & 0,7 & 0,06 & 0,09 \\
\hline VS113-3+M & 6,3 & 5,9 & 5,6 & 5,8 & 5,9 & 5,9 & 0,24 & 0,04 \\
\hline
\end{tabular}

table 2: 4,4'-MDA concentrations in polyol samples

Table 3 depicts the values of the MDA isomer distribution of the analyzed polyol samples. Due to the uncertainties in the isomer identification the concentration values of the isomers other than 4,4'-MDA were assigned to the isomers labeled A and B. Furthermore, the total MDA content was calculated:

\begin{tabular}{|l|c|c|r|r|}
\hline \multirow{2}{*}{ sample } & \multicolumn{5}{c|}{ CMDA [\%] } \\
\cline { 2 - 5 } & $4,4^{\prime}$ 'MDA & $\begin{array}{c}\text { MDA } \\
\text { isomer A }\end{array}$ & $\begin{array}{c}\text { MDA } \\
\text { isomer B }\end{array}$ & $\begin{array}{c}\text { total MDA } \\
\text { content }\end{array}$ \\
\hline VSA14 & 7,0 & 0,4 & 4,1 & $11,5 \pm 0,3$ \\
\hline VS14ACAC & 1,7 & 0,3 & 1,6 & $3,6 \pm 0,3$ \\
\hline VS127 & 6,0 & 0,3 & 3,7 & $10,0 \pm 0,4$ \\
\hline VSA & 4,5 & 0,3 & 3,0 & $7,8 \pm 0,4$ \\
\hline VSA+50 & 3,1 & 0,2 & 2,1 & $5,4 \pm 0,3$ \\
\hline VS113-1+GE & 0,7 & 0,2 & 1,0 & $1,9 \pm 0,2$ \\
\hline VS113-3+M & 5,9 & 0,4 & 3,8 & $10,1 \pm 0,4$ \\
\hline
\end{tabular}

table 3: MDA isomer distribution in polyol samples

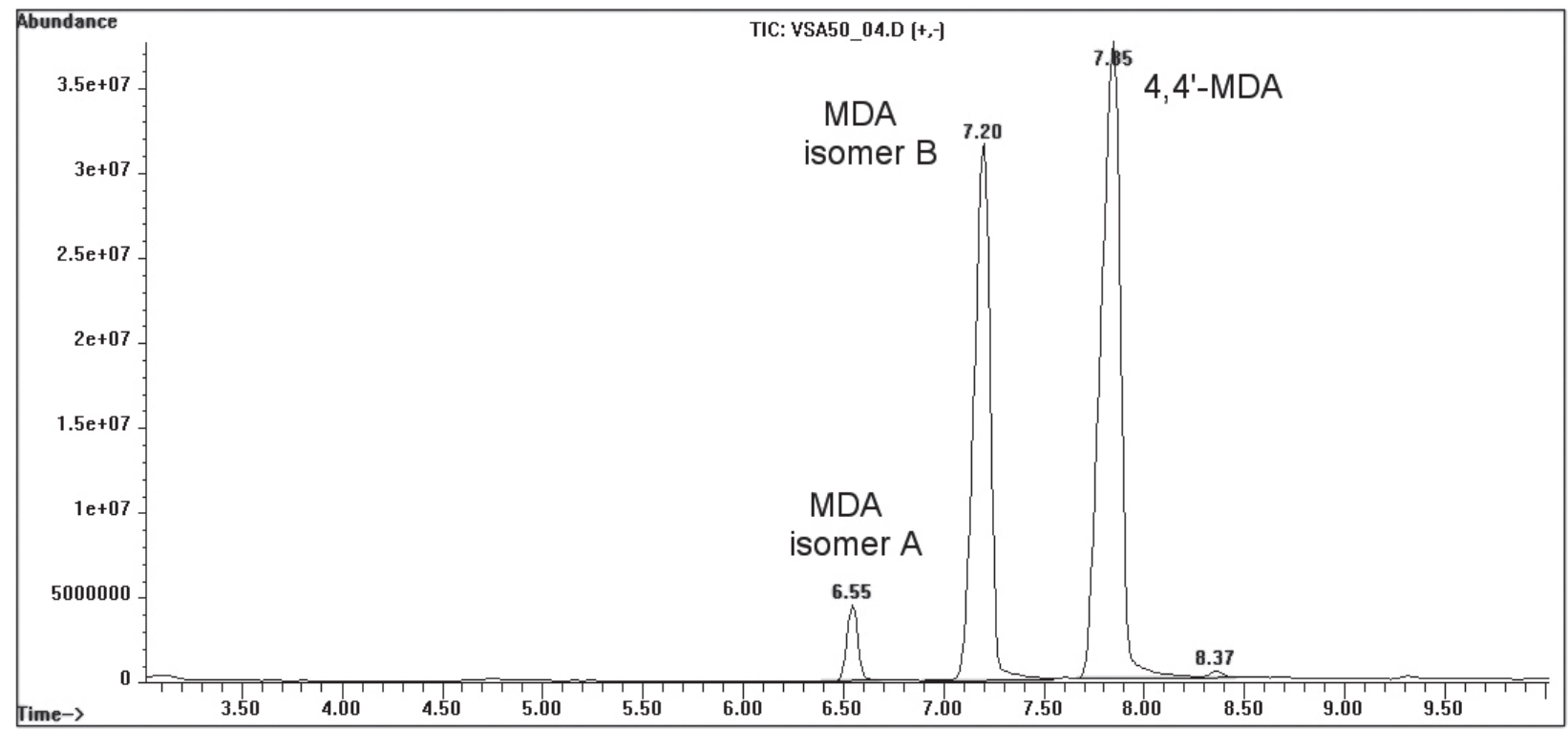

figure 4: gas chromatogram of the MDA containing fraction - sample VSA50 


\section{Discussion}

4.1 MDA concentrations in polyol samples and deamination

Considering the determined remarkable high MDA concentrations in the polyols derived from flexible PUR foam solvolysis up to $7 \%$ for $4,4^{\prime}$ '-MDA and $11.5 \%$ for the total amount of MDA isomers, it results that the hitherto existing concept of the MDA formation must be extended. According to the current theory the formation of MDA during the solvolysis of the polyurethanes essentially depends on the existence of water in the PUR material [1] (Behrendt et. al. 2009), but this would not explain the rather large amounts of MDA formed. It can be assumed, that the thermolysis of the urethane group, possibly as a catalyzed process, is the major source of the MDA formation [17] (Saunders 1967). To a full explanation further investigations have to be made.

Because of the high MDA concentrations detected it is necessary to implement a deamination treatment to the polyols. The deamination can be performed either by adding a deamination reagent like long chain glycidyl ethers to the solvolysis reaction mixture to transform the MDA just in the nascent state into less toxic amino alcohols [7] (Gassan et. al. 1992), or by carrying out the deamination as a second reaction step after the PUR solvolysis [4] (Thor et. al. 2005).

By way of example one of the analyzed polyol samples (VS113-1+GE) is a product of a subsequent deamination treatment with 2-ethyl-hexyl-glycidylether and has a 4,4'-MDA concentration of only $0.7 \%$. This is still much more than the legally allowed value, but could be further decreased by variations in technology or by increasing the added amount of glycidyl ether.

\subsection{Limits of the method}

The accuracy of the method is limited essentially by the observational error caused by fluctuations of the instrument precision, while the sensitivity of the method depends mainly on the amount of analyzable MDA after the SEC fractionation procedure. In general, uncertainties related to the sample handling by the instrument as well as to the instrument signal itself lead to higher coefficients of variation $\left(\mathrm{c}_{\mathrm{v}}\right)$ when the substance causing the signal response is present in lower amounts.

Regarding the GC/MS detection limit of ca. 2 ng 4,4'MDA per $\mu \mathrm{THF}$, which corresponds approximately to a value of $0.01 \% \mathrm{MDA}$ in a polyol sample before SEC, this leads in combination with the margins of error of both analytical steps to a limit of quantitation (LOQ) of about $0.04 \% \mathrm{MDA}$ in a polyol sample. To accurately determine the limit of detection and the limit of quantitation further injections followed by statistical calculations have to be made [16] (Hübschmann 2008).

\subsection{Improvement of the method}

Retaining the applied instrumental equipment the method itself could be improved by repeating the step of SEC fractionation several times for one sample to gain a higher amount of analyzable substance and/or by increasing the injection volume at the GC/MS.

\section{Conclusions}

The method developed to determine the concentration of 4,4'-diamino diphenyl methane (MDA) in polyols derived from PUR foam solvolysis proved to be applicable for MDA concentrations as low as $0.05 \%$ up to values greater than $10 \%$ MDA in polyol samples.

The hitherto existing concept of the MDA formation during the PUR solvolysis has to be extended to fully explain the high amounts of MDA determined.

To the polyols obtained by such a solvolysis process a deamination procedure has to be applied to decrease their remarkable high MDA content.

\section{References}

[1] Behrendt, G.; Naber, B. W. (2009): The chemical recycling of polyurethanes (review), Journal of the University of Chemical Technology and Metallurgy, 44, 1, 3-23, Sofia.

[2] Behrendt, G.; Stoychev, V.; Lehmann, D. (2005): Chemisches Recycling von Polyurethan-Blockweichschaumstoffen, in: Wiss. Beiträge 2005 der Techn. Fachhochschule Wildau, 85-92.

[3] Raßhofer, W. (1995): Das Recycling von Polyurethanen, CarlHanser-Verlag, München.

[4] Thor; Koch; Stuhr; Naber (2005): Verfahren zur kontinuierlichen Herstellung von Recyclatpolyolen aus Polyurethanen, DE-OS 10 2005038375.

[5] Parrinello; Thorpe; Verheist; Hopper; De Witte (ICI PLC) (1997): Recycling of Flexible Foam, USP 5,691,389.

[6] Kierkus, P. C.; You, K. K. (1998): Process for the preparation of recyclate polyols having a low amine content, USP 5,763,692.

[7] Gassan; Naber; Neiss; Moeckel; Weisflog (BASF Schwarzheide $\mathrm{GmbH}$ ) (1992): Preparation of Recyclate Polyols and the Use thereof in the Preparation of Polyurethanes, USP 5,357,006; DEOS 4235335 .

[8] Stoychev; Klockemann; Werner (2006): Verfahren und Vorrichtung zur Wiedergewinnung von Rohstoffen aus Polyurethanen, DE102006034613A1. 
[9] Skarping, G.; Dalene, M. (1995): Determination of 4,4'-methylenediphenyldianiline (MDA) and identification of isomers in technical-grade MDA in hydrolysed plasma and urine from workers exposed to methylene diphenyldiisocyanate by gas chromatography-mass spectrometry, Journal of Chromatography B. 663, 209-216.

[10] Neumeister, C. (NIOSH/DPSE) (1994): 4,4'-Methylenedianiline - HPLC, UV and Electrochemical Detection, NIOSH Manual of Analytical Methods (NMAM), Fourth Edition.

[11] Shintani, H.; Nakamura, A. (1989): Analysis of the carcinogen 4,4'-methylenedianiline (MDA) in gamma-ray and in autoclavesterilized polyurethane, Fresenius Z. Anal. Chem. 333:637-640.

[12] Mattrel, P.; Richter, K. (1995): Methode zur Bestimmung der Emission von Diphenylmethan-4,4'-diisocyanat (MDI) aus isocyanatgebundenen Holzwerkstoffen, Holz als Roh- und Werkstoff 53, 321-326@ Springer-Verlag.

[13] Mazzu, A.L.; Smith, C. P. (2004): Determination of extractable methylene dianiline in thermoplastic polyurethanes by HPLC, Journal of Biomedical Materials Research, Volume 18 Issue 8, 961968.

[14] Schmidt, T. C.; Haas, R. et al. (1997): Derivatization of aromatic amines for analysis in ammunition wastewater I. Derivatization via bromination of the aromatic ring, Fresenius' Journal of Analytical Chemistry, Volume 357, Number 7.

[15] Mori, S. (1999): Size Exclusion Chromatography, Springer-Verlag Berlin and Heidelberg.

[16] Hübschmann, H.-J. (2008): Handbook of GC/MS: Fundamentals and Applications, Wiley-VCH, 2nd ed.

[17] Saunders, J. H. (1967): Thermal Degradation and Flammability of Polyurethane Foams, Nat. Acad. Sci. Publ. No. 1462, Washington, p. 123.

[18] WHO, IARC Monographs 57 (1993), 39 (1985).

[19] REGULATION (EC) No 1272/2008 OF THE EUROPEAN PARLIAMENT AND OF THE COUNCIL of December 2008 on classification, labeling and packaging of substances and mixtures.

\section{Authors}

\section{Dipl.-Chemiker Eckhart Kornejew}

TH Wildau [FH]

Fachbereich Ingenieurwesen/Wirtschaftsingenieurwesen

Tel. +49 3375 508-296

eckhart.kornejew@tfh-wildau.de

Diplom-Ingenieur Valentin Stoychev

TH Wildau [FH]

Fachbereich Ingenieurwesen/Wirtschaftsingenieurwesen

Tel. +49 3375 508-338

valentin.stoychev@tfh-wildau.de 